\title{
Is CYP2D6 phenotype predictable from CYP2D6 genotype?
}

Ádám Ferenc Kiss ${ }^{\mathrm{a}}$, Katalin Tóth ${ }^{\mathrm{a}}$, Cintia Juhász ${ }^{\mathrm{a}}$, Manna Temesvári ${ }^{\mathrm{a}}$, József Paulik ${ }^{\mathrm{b}}$, Gábor Hirka $^{\mathrm{c}}$, Katalin Monostory ${ }^{\mathrm{a}}$

${ }^{a}$ Research Centre for Natural Sciences, Hungarian Academy of Sciences, Magyar tudósok 2, H-1117 Budapest, Hungary

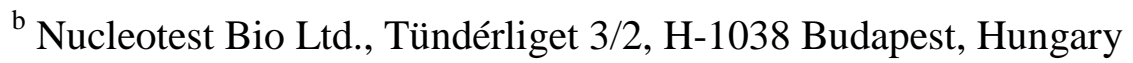

c Toxi-Coop Toxicological Research Center Co., Magyar jakobinusok 4, H-1122 Budapest, Hungary

\section{Highlights}

- CYP2D6 genotype-based phenotype estimation is of particular importance to personalized medication strategy.

- The non-sequencing genotyping platform identified the most frequent CYP2D6 allelic variants (in $67 \%$ of the donors).

- The gain-of-function mutation $(-1584 C>G)$ could explain the underestimation of CYP2D6 genotype-based prediction.

- Some rare loss-of-function mutations that were not captured, could result in overestimation of CYP2D6 activity prediction.

- CYP2D6 phenotype overestimation may also come from external factors modifying CYP2D6 activity or altering hepatic function.

- A comprehensive genotyping and consideration of phenoconversion can improve the genotype-based CYP2D6 phenotype prediction. 


\section{Abstract}

Genetic polymorphism of cytochrome P450s results in clinically significant modifications in patients' drug metabolizing capacities. CYP2D6 has a crucial role in the elimination of several clinically important drugs (antiarrhythmics, beta-adrenergic blockers, psychopharmacons and analgesics); however, the prediction of the phenotypic appearance of CYP2D6 is a challenge. Since single nucleotide polymorphisms and gene copy number variations (gene deletion and multiplication) frequently occur in CYP2D6 gene, CYP2D6 activity particularly depends on the genetic factors.

Microsomal CYP2D6 activities (dextromethorphan O-demethylation) and CYP2D6 genotypes for the most frequent allelic variants (CYP2D6*3, *4, *5, *6, *10,*41 and duplication) were determined in 128 human liver samples derived from Hungarian organ donors. Substantial inter-individual variations were observed in CYP2D6 metabolic activities that were successfully predicted from the CYP2D6 genotypes in $67 \%$ of the donors. The underestimation of CYP2D6 phenotypes in $12.5 \%$ of the donors was assumed to be originated from the overlapping ranges of CYP2D6 activity among similar diplotypes or from the presence of $-1584 C>G$ in the promoter region evoking increased transcription of the wild-type CYP2D6 allele. In an appreciable number of donors (20.3\%), the genotype-based CYP2D6 phenotype prediction was overestimated because of the rare CYP2D6 allelic variants which were not included in our genotyping platform or some external factors that could alter CYP2D6 activity (medication with CYP2D6 substrate/inhibitor) and hepatic function (Augmentin therapy, chronic alcohol consumption).

In conclusion, CYP2D6 genotyping for the most frequent allelic variants was able to reliably predict CYP2D6 phenotypes in most donors; however, the external factors modifying the phenotypic appearance of CYP2D6 had to be taken into account. Personalized medication strategy should include monitoring of CYP2D6 genotype in a more comprehensive manner 
and should take external factors into consideration for an appropriate prediction of CYP2D6 metabolizing capacity.

Keywords: CYP2D6 genotype, genetic polymorphism, phenotype prediction, personalized medication

Abbreviations: CNV, copy number variation; CYP, cytochrome P450; EM, extensive metabolizer; IM, intermediate metabolizer; PCR, polymerase chain reaction; PM, poor metabolizer; SNP, single nucleotide polymorphism; UM, ultra-rapid metabolizer 


\section{Introduction}

Most drugs show inter-individual differences in their responses and efficacy due to the substantial variability of the capacity of drug metabolizing enzymes between individuals. The primary drug metabolizing enzymes belong to the cytochrome P450 (CYP) superfamily, which are responsible for the metabolism of more than 90 percent of the drugs on the market. In mammalian species, CYP enzymes are present in most tissues (e.g. gut wall, kidney, brain, lung); however, they are mainly expressed in the liver. Most of them are highly polymorphic resulting in clinically significant modifications in drug metabolizing capacities. The major genetic polymorphisms influencing drug metabolizing activity are related to the metabolism by CYP2D6. Although CYP2D6 presents only a small proportion of hepatic CYP enzymes (2\%), it is one of the most important drug metabolizing enzymes, catalysing the metabolism of approximately 25\% of the drugs on the market. CYP2D6 is involved in the biotransformation of numerous clinically used drugs, such as beta-blockers (metoprolol, bufuralol, propranolol), opioids (codeine, tramadol), antidepressants (amitriptyline, imipramine, fluoxetine, mianserin, venlafaxine), antipsychotics (aripiprazole, haloperidol, olanzapine, risperidone), antitussives (dextromethorphan) and anticancer drugs (tamoxifen) [1].

The CYP2D6 gene is located on chromosome 22q13.1 nearby two homologous, non-functional pseudogenes, CYP2D7P and CYP2D8P [2]. CYP2D6 gene is highly polymorphic; hitherto more than one hundred allelic variants were identified, a number of them containing mutations that have clinically relevant impact (http://www.cypalleles.ki.se/cyp2d6.htm) [34]. Both single nucleotide polymorphisms (SNP) and gene copy number variations (CNV) (such as gene deletion and duplication/multiplication) frequently occur. Among the allelic variants, there are fully functional alleles (e.g. *1, *2), alleles with reduced function (e.g. *9, *10,*41) and null (non- 
functional) alleles (e.g. *3, *4, *5, *6). Duplication of the functional allele leads to increased gene expression and enzyme activity $[3,4]$. Due to the wide range of CYP2D6 enzyme activity within the population, the patients can be divided into four metabolizer groups: poor (PM), intermediate (IM), extensive (EM) and ultra-rapid metabolizers (UM) [5]. Since CYP2D6 gene expression is not inducible by xenobiotics, genetic factors are thought to play a substantial role in the phenotypic appearance of CYP2D6.

Typically, subjects considered to be PMs carry two non-functional alleles which do not encode a functional protein product; thus, they do not display detectable enzyme activity. Several studies established clinical risks in the case of PM patients treated with CYP2D6 substrate drugs [5-7]. The most frequent non-functional allelic variant is CYP2D6*4 in Caucasians (European white population) with an allele frequency of $18-23 \%$ [8]. CYP2D6*3 and $* 6$ allelic variants, which occur at allele frequency of about $1 \%$ in Caucasians, contain one nucleotide deletion causing frame-shift mutation and the lack of functional CYP2D6 protein. CYP2D6*5, which occurs in $2-5 \%$ of Caucasians, means the deletion of the entire gene and the lack of CYP2D6 enzyme expression [9]. The alleles CYP2D6*10 and *41 give rise to significantly decreased enzyme activity. However, the allele frequency of $C Y P 2 D 6^{*} 10$ is $1-2 \%$ in Caucasians, this allele variant is typical in the Asian population with an allele frequency of $33-43 \%$ [10]. CYP2D6*10 has a 100C $>$ T SNP leading to the instability of the enzyme protein [11]. CYP2D6*41 contains an intronic 2988G>A SNP causing increased levels of a non-functional splice variant and accordingly lower CYP2D6 metabolic activity [12]. CYP2D6*41 frequently occurs in Caucasians (allele frequency: 7-10\%) [13]. UM phenotype has also clinical relevance, since high enzyme activity can be responsible for adverse drug effects or for therapeutic failure depending on the used drug. Primarily, duplication or multiplication of the functional CYP2D6 gene accounts for UM phenotype; however, copy number increase does not explain all cases of UMs [14]. 
Up to now, several in vivo and in vitro studies were implemented to investigate an applicable formula with which certain CYP2D6 genotype could be translated to the corresponding phenotype. For example, Gaedigk et al. [15] introduced an activity score system, which assigns a number for each allelic variant depending on the functionality of the allele. Although more investigations were achieved at in vivo conditions to evaluate the effect of CYP2D6 polymorphism directly on the enzyme activity, human derived systems, such as human liver microsomes, are recommended to be used. Incubation of the microsomes with CYP-specific substrates provides direct information about the specific CYP enzyme activity.

Due to the clinical significance of the medications metabolized by CYP2D6, it would be important to obtain accurate estimation of CYP2D6 activity based on a fast and simple genotyping method. The aim of the present work was to investigate the accuracy of CYP2D6 phenotype prediction from genotype and the reliability of CYP2D6 genotype interpretation. Human liver microsomes were used to evaluate CYP2D6 metabolic activity, and the samples were genotyped for the most frequent allelic variants in the Caucasian population.

\section{Materials and methods}

\subsection{Human liver microsomes}

Human liver tissues $(\mathrm{N}=128)$ were obtained from organ transplant donors at the Department of Transplantation and Surgery, Semmelweis University (Budapest, Hungary). Permission of the Hungarian Committee of Science and Research Ethics was obtained to use human tissues. Clinical histories of the donors are shown in Table 1. Human livers were perfused with Euro-Collin’s solution (Fresenius AG, Bad Homburg vdH, Germany) and excised. The tissues were homogenized in $0.1 \mathrm{M}$ Tris-HCl buffer (pH 7.4) containing $1 \mathrm{mM}$ EDTA and $154 \mathrm{mM}$ 
KCl. Microsomes were prepared by differential centrifugation as described by van der Hoeven and Coon [16]. All procedures of preparation were performed at $4^{\circ} \mathrm{C}$. Microsomal protein content was determined by the method of Lowry et al. [17] with bovine serum albumin as the standard.

\subsection{CYP2D6 enzyme assay}

Published method was followed to determine dextromethorphan O-demethylation selective for CYP2D6 enzyme [18]. The incubation mixture contained NADPH-generating system (1 mM NADPH, $10 \mathrm{mM}$ glucose 6-phosphate, $5 \mathrm{mM} \mathrm{MgCl}_{2}$ and 2 units/ml glucose 6-phosphate dehydrogenase), human liver microsomes $(0.8 \mathrm{mg} / \mathrm{ml})$ and the CYP2D6 selective substrate, dextromethorphan (1 mM). After a 20-min incubation, the reaction was terminated by ice-cold methanol and the incubation mixture was centrifuged for $10 \mathrm{~min}$ at $10.000 \times \mathrm{g}$. The supernatant was analysed for the concentration of dextromethorphan and its metabolite

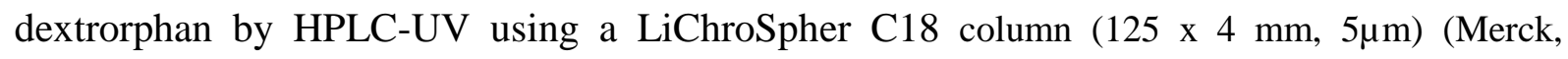
Darmstadt, Germany). The mobile phase consisted of $1 \mathrm{mM}$ Na-perchlorate (pH 2.6) and acetonitrile $(71: 29 \mathrm{v} / \mathrm{v})$. The rates of enzyme activity were linearly dependent upon the amount of microsomal protein added for the incubation period. CYP2D6 enzyme assay for each donor was performed in triplicate and median (min, max) was calculated.

\subsection{CYP2D6 genotyping}

Hydrolysis single nucleotide polymorphism (SNP) analysis for CYP2D6*3, CYP2D6*4, CYP2D6*6, CYP2D6*10 and CYP2D6*41 was performed by polymerase chain reaction (PCR) with TaqMan probes (Metabion, Planegg/Steinkirchen, Germany) using a CFX96 real-time detection system (Bio-Rad Laboratories). Primers and probes (Table 2) were designed based on the reference SNP sequences in the National Center for Biotechnology Information reference assembly. Genomic DNA was isolated from human liver samples by Quick-DNA $^{\text {TM }}$ Universal Kit (Zymo Research, Irvine, CA). PCR was carried out with 30 ng 
of genomic DNA by using Luminaris Color Probe qPCR Master Mix (Thermo Fisher Scientific, Waltham, MA). Allelic discrimination was based on the design of two TaqMan probes, specific for the wild type allele and the mutant allele labelled with different fluorescent tags (FAM, CalRed610 or HEX). The corresponding CYP2D6 genotypes were distinguished by plotting the relative fluorescent values for wild type and mutant alleles, hereby three allelic clusters were determined: homozygous wild type, homozygous mutant and heterozygous genotype. To confirm the results of CYP2D6 genotyping, a sequence analysis was also performed. One hundred nanograms of DNA were amplified by using the primers designed for the hydrolysis SNP analysis and the Luminaris Color Probe qPCR Master Mix. The PCR products were sequenced directly in an ABI PRISM 3100 Genetic Analyzer (Applied Biosystems, Foster City, CA) by the Sequencing Service of Biomi Ltd. (Gödöllö, Hungary).

To access CYP2D6 gene copy number, TaqMan ${ }^{\circledR}$ Copy Number Assay (assay id: Hs00010001_cn) was purchased from Thermo Fisher Scientific (Waltham, MA). To distinguish duplication of CYP2D6*1 allele and CYP2D6*4 allele, a real-time PCR method was developed combining quantitative PCR and genotyping PCR with TaqMan probes. Human RNase $P$ was applied as the reference gene using TaqMan ${ }^{\circledR}$ Copy Number Reference Assay (Thermo Fisher Scientific, Waltham, MA). CYP2D6*4 genotyping and RNase P reference assay were performed parallel, then relative copies of the wild type (CYP2D6*1) and CYP2D6*4 alleles were obtained based on the relative quantity of each alleles compared to a control sample carrying exactly one wild type allele and one CYP2D6*4 allele. $20 \mathrm{ng}$ of genomic DNA was used in a finale volume of $20 \mu \mathrm{l}$ reaction and the PCR conditions were as follows: $50^{\circ} \mathrm{C}$ for $2 \mathrm{~min}, 95^{\circ} \mathrm{C}$ for $10 \mathrm{~min}$, then 40 cycles of $95^{\circ} \mathrm{C}$ for $15 \mathrm{~s}$ and $55^{\circ} \mathrm{C}$ for $60 \mathrm{~s}$. Each sample was run in triplicate. Relative quantification of copy numbers was performed using CFX Manager Software Version 3.1 (Bio-Rad Laboratories). 


\subsection{Data analysis}

Hepatic CYP2D6 activities were determined individually in each donor, the frequency distribution of the CYP2D6 activities were recorded for 128 donors, and four categories (low, intermediate, high, ultra-high) were distinguished for poor, intermediate, extensive and ultra-rapid metabolizers. Approximately $10-15 \%$ of the donors displaying low CYP2D6 activity ( $<95-100 \mathrm{pmol} /[\mathrm{mg}$ protein*min]) were designated as poor metabolizers, whereas $10-15 \%$ of the donors with ultra-high activities (>720 pmol/[mg protein*min]) were designated as ultra-rapid metabolizers. The donors displaying CYP2D6 activities between 100 and $720 \mathrm{pmol} /(\mathrm{mg}$ protein*min) were considered to be intermediate and extensive metabolizers. The cutoff value between intermediate and extensive metabolizers was the median of CYP2D6 activities (290 pmol/[mg protein*min]). The donors' CYP2D6 phenotypes were also predicted from their CYP2D6 genotypes, and the accuracy of prediction was evaluated on the basis of the activity-based CYP2D6 categories. The donors carrying two loss-of-function or reduced-function alleles $(* 3, * 4, * 5, * 6, * 10, * 41)$ were considered to be poor metabolizers, those with one functional $(* 1)$ and one non-functional alleles were designated as intermediate metabolizers, the subjects with two functional alleles $(C Y P 2 D 6 * 1 / * 1)$ were extensive metabolizers, whereas the donors carrying duplicated or multiplicated functional alleles $(C Y P 2 D 6 * 1 \times 2$ or $C Y P 2 D 6 * 1 \times N)$ were ultra-rapid metabolizers.

\section{Results}

\subsection{Hepatic CYP2D6 activities}

CYP2D6 metabolic activities were determined in hepatic microsomal fractions of 128 Hungarian deceased donors. Dextromethorphan was used as a substrate selective for CYP2D6, and the formation of dextrorphan was quantified based on „per mg of microsomal 
protein and per minute”. The frequency distribution of CYP2D6 activities in the Hungarian organ donors is shown on Figure 1. Inter-individual variations of CYP2D6 activities ranged from non-detectable to rather high values and the activity values did not show a Gaussian distribution. The cutoff values $(\mathrm{CI}=95 \%)$ between the categories of poor, intermediate, extensive and ultra-rapid metabolizers were 95-100, 290 and 720 pmol/(mg protein*min), respectively (see 2.4).

The inter-individual variations of CYP2D6 activity were not influenced by the donors' demographic parameters, such as sex or age (data not shown). In most cases, the death of the donors was caused by cerebral hemorrhage/hematoma (62/128) or accident (30/128); however, the incidence of stroke and tumour was much less (7/128 and 16/128, respectively). Comparing the groups of donors categorized according to the cause of death, we found no significant differences in CYP2D6 metabolic activities among the groups (data not shown). Chronic alcohol consumption was diagnosed at 11 donors as well as four and two of the 128 subjects received CYP2D6-substrates/inhibitors (beta-adrenergic blockers, metoprolol, haloperidol) and Augmentin (amoxicillin and clavulanic acid), respectively.

\subsection{The donors’ CYP2D6 genotypes}

CYP2D6 genotype-based phenotype prediction was performed for each donor by determining the allelic variants most frequently occurring in Caucasian populations. In Hungarian organ donors, the most frequent non-functional allele was $C Y P 2 D 6 * 4$, whereas $C Y P 2 D 6 * 3$, *5 and *6 occurred only in 2, 2 and 7 of 128 donors, respectively (Table 3). Particularly, the prevalence of the reduced function CYP2D6*41 allele was $7.8 \%$, whereas CYP2D6*10 allele had lower occurrence in our cohort (3.5\%). Interestingly, the duplication/multiplication of the CYP2D6 gene in 128 donors was extremely frequent (19.9\%), in contrast to the duplication frequencies previously reported in Caucasians (2-5\%). Rideg et al. [19] have reported 
CYP2D6 gene duplication with $1.8 \%$ frequency in Hungarian population $(\mathrm{N}=112)$, similarly to the findings in other European cohorts [8, 15, 20, 33]. Wild-type allele (CYP2D6*1) was identified when none of the allelic variants (CYP2D6*3, *4, *5, *6, *10, *41 or duplication) was detected. $-1584 C>G$ SNP contributing to increased expression of CYP2D6 gene (Dorado et al. [21], Llerena et al. [22]), was also assayed in donors who carried $C Y P 2 D 6 * 1 / * 1$ genotype to find associations between the mutation in the promoter region of CYP2D6 gene and UM phenotype.

Duplication of the non-functional alleles, including the highly frequent CYP2D6*4 allele can also occur; however, such arrangements do not increase CYP2D6 activity. Copy number determination by TaqMan Copy Number Assay (Thermo Fisher Scientific) gives insufficient information about the copy number of different allelic variants in case of a heterozygous CYP2D6*1/*4 individual, since this assay cannot distinguish the duplication of the wild-type alleles $(* 1 \times 2 / * 4)$ and non-functional alleles $(* 1 / * 4 \times 2)$. We developed a novel fast and simple method combining quantitative PCR and genotyping PCR with TaqMan probes (see 2.3), whereby allele-specific copy number determination was successfully implemented on donors who were heterozygous for CYP2D6*4 allele.

\subsection{Association between dextromethorphan O-demethylase activity and CYP2D6 genotype}

CYP2D6 phenotypes of the 128 organ donors were predicted from their CYP2D6 genotypes categorizing them as poor, intermediate, extensive and ultra-rapid metabolizers according to the presence or absence of allelic variants with altered functionality. CYP2D6 genotype groups with the corresponding metabolic activities are shown in Figure 2. All the donors who had no wild type allele (CYP2D6*1) showed low CYP2D6 activity; however, we did not find differences in dextromethorphan O-demethylation activity between the donors with two non-functional alleles and those with one non-functional $(* 3, * 4, * 5$ or $* 6$ ) and one allele associated with reduced CYP2D6 function $(* 10$ or $* 41)$. The donors with only one wild type 
allele were considered to be IMs; however, due to the reduced activity attributed to CYP2D6*10 or CYP2D6*41 alleles, we were unable to define a strict border-line between IM and EM phenotype groups. Duplication of non-functional alleles has no effect on CYP2D6 activity, thus $C Y P 2 D 6 * 1 / * 4 \times 2$ and $C Y P 2 D 6 * 1 / * 4$ genotypes were considered to be identical in respect of the CYP2D6 activity. Two CYP2D6 genotype groups were considered to be EMs: 1) donors carrying CYP2D6*1/*1 and 2) subjects with duplicated wild type allele and one non-functional allele or allele variant with decreased function. These EM individuals with two functional alleles occurred in $45 \%$ of the organ donors. Although only two functional alleles were identified, seven donors displayed UM phenotype (dextromethorphan O-demethylation $>720 \mathrm{pmol} /[\mathrm{mg}$ protein*min $]$ ). Two individuals with $C Y P 2 D 6 * 1 / * 1$ genotype had extremely high CYP2D6 activity (1242 and 1461 pmol/[mg protein*min]), which was attributed to the heterozygous (CG) and the homozygous (GG) genotypes at the position of -1584 . On the other hand, we found several donors (12/128) carrying two wild type alleles (CYP2D6*1/*1) whose hepatic microsomes displayed significantly lower activity than it could be predicted from the corresponding CYP2D6 genotype. Among these individuals, the anamnesis of four of them indicated chronic alcohol consumption, two were under CYP2D6 substrate or inhibitor therapy (beta adrenergic blockers, haloperidol), and one subject received Augmentin. The combination of amoxicillin and clavulanic acid in Augmentin has been reported to evoke liver dysfunction including reduced CYP activities [23]. UM phenotype is primarily attributed to CYP2D6 gene duplication/multiplication; however, of 13 subjects with ultra-rapid CYP2D6 activity (>720 pmol/[mg protein*min]), we found only 4 donors who carried more than two functional alleles (CYP2D6*1/*1x2).

In conclusion, of 128 organ donors, the CYP2D6 phenotypes of 86 were successfully predicted from the genotypes. This means that the accuracy of prediction was 67\%. CYP2D6 phenotypes of 16 individuals were underestimated, and the elevated CYP2D6 activities in 5 
donors could be explained with $-1584 C>G$ SNP, resulting in increased transcription of CYP2D6*1 allele [21, 22]. In 26 subjects, the phenotype was overestimated. Several individuals had PM phenotype (dextromethorphan O-demethylation activity lower than 100 $\mathrm{pmol} /[\mathrm{mg}$ protein*min]), despite the presence of two or more wild type alleles. External factors, such as CYP2D6 substrate or inhibitor therapy, chronic alcohol consumption and Augmentin therapy were observed in 17 of the 128 donors, which resulted in overestimation of CYP2D6 activity.

\section{Discussion}

CYP2D6 has a crucial role in the metabolism of several clinically important drugs; thus, any changes in CYP2D6 activity influence the patient's response to a drug primarily metabolized by CYP2D6. According to the present knowledge, the expression of CYP2D6 gene is not inducible by any known xenobiotics in contrast to other drug metabolizing CYP enzymes [24, 25]. Since CYP2D6 gene is remarkably polymorphic, the main factor that determines the enzyme activity is thought to be the genetic variations. Personalized medication requires a reliable method to predict CYP2D6 activity from patients' genotype for a long while. Due to the numerous allelic variants, it is a challenge to elaborate an applicable method for CYP2D6 phenotype prediction. Human liver cellular and subcellular systems, such as hepatocytes and microsomes, seem to be a good platform to investigate CYP2D6 genotype-phenotype associations. In this study, CYP2D6 metabolic activities were determined in human liver microsomes derived from Hungarian organ donors using dextromethorphan selective for CYP2D6. The liver samples were CYP2D6-genotyped for the most frequent allelic variants occurring in Caucasian populations. The allele frequencies for CYP2D6*3, *4, *5, *6, *10 and $* 41$ were concordant with previously reported data except for the prevalence of gene duplications (Table 3) [9, 24], which was substantially higher (19.9\%) than it was reported in Caucasian populations (2-5\%). We were unable to correlate the elevated frequency of 
CYP2D6 duplication to any available clinical information about the donors. An association between CYP2D6 duplication and the cause of donors' death may be assumed. Interestingly, the outstanding frequency of duplication was unequivocally observed in donors died because of cerebral hemorrhage (26\%) or accident (33\%). It should be emphasized that the most frequent causes of death were cerebral hemorrhage and accident (92/128). Nevertheless, elevated frequency of duplication was not observed among donors died owing to stroke (6\%) or tumour (9\%). Regarding to the location of the hemorrhage, the cerebral hemorrhage was mostly diagnosed as subarachnoidal hemorrhage/hematoma. Unfortunately, in terms of donors who died in accident, we had limited information about the circumstances; most of them were involved in car, motor or bike accident, however, some suggestive facts, such as suicide, asphyxia and seizure, were also listed as causes of fatal injuries.

CYP2D6 phenotype prediction was based on the determination of the most frequent allelic variants occurring in Caucasians. The donors with two non-functional alleles displayed low CYP2D6 metabolic activity; albeit in some cases the formation rate of the metabolite dextrorphan was in the activity range of IM. The donors with one allele associated with reduced activity (CYP2D6*10 or *41) and one non-functional allele (CYP2D6*3, *4, *5 or *6) appear to display some activities resulting in poor or intermediate metabolizer phenotypes. In agreement with the preceding interpretation by Hicks et al. [26] and Gaedigk [27], the range of metabolic activity among similar CYP2D6 diplotypes can cover even two phenotype categories. Therefore making distinction between the subjects carrying two non-functional alleles and those with one non-functional and one reduced-function alleles is unnecessary. Since both genotypes result in low CYP2D6 activities, the strategy of personalized medication for such patients should take the dose reduction of CYP2D6 substrates into consideration. 
The presence of one functional $\left(C Y P 2 D 6^{*} 1\right)$ and one non-functional alleles is considered to be associated with IM phenotype, and the genotype-based prediction for donors' phenotype was correct in most subjects (17/22). Although the microsomal CYP2D6 activities of three donors were in EM activity range despite one functional allele, each of them carried the $-1584 C>G$ SNP, which was reported to evoke increased CYP2D6 expression [21, 22]. The presence of one functional $(C Y P 2 D 6 * 1)$ and one reduced-function $(C Y P 2 D 6 * 10$ or *41) alleles can also be predicted to be translated to IM phenotype; however, CYP2D6*1/*10 or CYP2D6*1/*41 genotypes resulted in slightly increased CYP2D6 activities, reassigning these donors to EM activity group. It could be explained by the reduced function of CYP2D6*10 and $* 41$ allele variants, which entails a broader range of CYP2D6 activity spanning IM and EM phenotype categories [26, 27].

CYP2D6*1/*1 genotype, predicted to lead to EM phenotype, was identified in 36 donors; however, only 20 displayed dextromethorphan O-demethylation activity in the EM activity range and 16 were out of this range. In two of the $C Y P 2 D 6^{*} 1 / * 1$ donors, exhibiting the highest CYP2D6 activities, $C>G$ SNP was detected at the position of -1584 (one was homozygous $G / G$, the other was $C / G$ heterozygous), reassigning them to UM category. Twelve individuals showed significantly lower activities than it would be predicted from the CYP2D6*1/*1 genotype. More than half of the donors with $C Y P 2 D 6 * 1 / * 1 \times 2$ genotype were also demonstrated to belong to the PM, IM or EM activity-based categories, although these individuals had more than two copies of the wild type allele that would designate UM phenotype.

CYP2D6 genotype-based phenotype prediction overestimated CYP2D6 activity of 26 donors which can be explained by 1) the non-sequencing genotype platform we applied or 2) by some external factors that can modify the enzyme activity. Our non-sequencing genotyping approaches focused on some key polymorphisms that frequently occur in Caucasian 
populations; thus, some rare loss-of-function or reduced-function variants were not captured. The phenotype overestimation due to some external factors influencing CYP2D6 enzyme activity has also been indicated by several authors [28-30]. The impact of CYP2D6 substrates or inhibitors, such as beta adrenergic blockers or antipsychotics (haloperidol, risperidone, aripiprazole) on CYP2D6 activity is obvious. The anamnesis of some donors indicated chronic treatment with beta adrenergic blockers or haloperidol, thus, our results could confirm that the genotype-predicted UM and EM donors were simply converted into IMs or PMs by these substrates/inhibitors. Phenotype conversion can also be attributed to some aspecific agents, such as Augmentin treatment or chronic alcohol consumption. Augmentin is an antibiotic drug containing the combination of amoxycillin and clavulanic acid. The hepatotoxic effect of both Augmentin and alcohol has been well described [23, 31, 32]; however, their impact on CYP2D6 is hardly investigated. Our results suggested that Augmentin or chronic alcohol exposure of the donors might contribute to the reduction of CYP2D6 activity, leading to the inconsequence of the CYP2D6 genotype-based phenotype prediction. Because of the hepatotoxic exposure, eleven subjects were found to be PMs or IMs despite the presence of two or more CYP2D6*1 alleles. In addition, differences in CYP3A4 expression and activity among the donors could have a minor role in the observed disparities between genotype and phenotype, since at a milimolar substrate concentration, the contribution of CYP3A4 to the catalysis of dextromethorphan O-demethylation increases [33].

In conclusion, the phenotype prediction from CYP2D6 genotype was acceptable for $67 \%$ of the donors (86/128). The CYP2D6 activity of some donors $(12.5 \%)$ was found to be somewhat higher than it would be predicted from their CYP2D6 genotypes. The underestimation was assumed to be originated from 1) the overlapping ranges of CYP2D6 activity among similar diplotypes or 2) the presence of $C>G$ at the position of -1584 evoking 
increased transcription of certain CYP2D6 alleles. Although the $-1584 C>G$ SNP may account for the underestimation of CYP2D6 phenotype for some donors, the association between the $-1584 G$ allele and increased CYP2D6 activity requires further investigation. In an appreciable number of donors (20.3\%), the genotype-based CYP2D6 phenotype prediction was overestimated because of the rare CYP2D6 allelic variants which were not included in our genotyping platform or because of the possible external factors that could alter CYP2D6 activity and hepatic function. These findings suggested that although CYP2D6 genotyping for the most frequent allelic variants successfully predicted the phenotype in more than two thirds of the donors, some external factors could modify the phenotypic appearance of CYP2D6.

\section{Acknowledgement}

The authors are grateful to Ulrich Zanger (Dr Margarete Fischer-Bosch-Institute of Clinical Pharmacology, Stuttgart, Germany) for providing positive control samples with various CYP2D6 genotypes. This work was supported by the grant from the Hungarian Research Fund (OTKA K104459). 


\section{References}

[1] S.F. Zhou, Polymorphism of human cytochrome P450 2D6 and its clinical significance: Part I, Clinical pharmacokinetics, 48(11) (2009) 689-723

[2] S. Kimura, M. Umeno, R.C. Skoda, U.A. Meyer, F.J. Gonzalez, The human debrisoquine 4-hydroxylase (CYP2D) locus: sequence and identification of the polymorphic CYP2D6 gene, a related gene, and a pseudogene, Am. J. Hum. Genet, 45 (1989) 889-904

[3] M.L. Dahl, I. Johansson, L. Bertilsson, M. Ingelman-Sundberg, F. Sjöqvist, Ultrarapid hydroxylation of debrisoquine in a Swedish population: analysis of the molecular genetic basis, J. Pharmacol. Exp. Ther. 274 (1995) 516-520

[4] P. Dalén, M.L.Dahl, M.L. Bernal Ruiz, J. Nordin, L. Bertilsson, 10-hydroxylation of nortriptyline in white persons with $0,1,2,3$, and 13 functional CYP2D6 genes, Clinical Pharmacology \& Therapeutics, 63 (1998) 444-452

[5] U.M. Zanger, S. Raimundo, M. Eichelbaum, Cytochrome P450 2D6: overview and update on pharmacology, genetics, biochemistry, Naunyn-Schmiedeberg's archives of pharmacology, 369(1) (2004) 23-37

[6] J. Brockmoller, J. Kirchheiner, J. Schmider, S. Walter, C. Sachse, B. MullerOerlinghausen, I. Roots, The impact of the CYP2D6 polymorphism on haloperidol pharmacokinetics and on the outcome of haloperidol treatment, Clin Pharmacol Ther 72 (2002) 438-452

[7] H. Wuttke, T. Rau, R. Heide, K. Bergmann, M. Bohm, J. Weil, D. Werner, T. Eschenhagen, Increased frequency of cytochrome P450 2D6 poor metabolizers among patients with metoprolol-associated adverse effects, Clin. Pharmacol. Ther., 72 (2002) 429437

[8] M.L. Barclay, S.M. Sawyers, E.J. Begg, M Zhang, R.L. Roberts, M.A. Kennedy, J.M. Elliott, Correlation of CYP2D6 genotype with perhexiline phenotypic metabolizer status, Pharmacogenetics, 13 (2003) 627-632

[9] C. Sachse, J. Brockmoller, S. Bauer, I. Roots, Cytochrome P450 2D6 variants in a Caucasian population: allele frequencies and phenotypic consequences, Am. J. Hum. Genet., 60 (1997) 284-295

[10] D.R. Nelson, D.C. Zeldin, S.M. Hoffman, L.J. Maltais, H.M. Wain, D.W. Nebert, Comparison of cytochrome P450 (CYP) genes from the mouse and human genomes, including nomenclature recommendations for genes, pseudogenes and alternative-splice variants, Pharmacogenetics, 14 (2004) 1-18

[11] L.D. Bradford, CYP2D6 allele frequency in European Caucasians, Asians, Africans and their descendants, Pharmacogenomics, 3 (2002) 229-243

[12] T. Fukuda, Y. Nishida, S. Imaoka,T. Hiroi, M. Naohara, Y. Funae, J. Azuma, The decreased in vivo clearance of CYP2D6 substrates by CYP2D6*10 might be caused not only by the lowexpression but also by low affinity of CYP2D6, Arch. Biochem. Biophys., 380 (2000) 303-308

[13] C. Toscano, K. Klein, J. Blievernicht, E. Schaeffeler, T. Saussele, S. Raimundo, M. Eichelbaum, M. Schwab, U.M. Zanger, Impaired expression of CYP2D6 in intermediate metabolizers carrying the *41 allele caused by the intronic SNP 2988G>A: evidence for modulation of splicing events, Pharmacogenet. Genomics, 16 (2006) 755-766 
[14] S. Raimundo, C. Toscano, K. Klein,J. Fischer, E.U. Griese, M. Eichelbaum, M. Schwab, U.M. Zanger, A novel intronic mutation, 2988G4A, with high predictivity for impaired function of cytochrome P450 2D6 in white subjects, Clin. Pharmacol. Ther., 76 (2004) 128138

[15] A. Gaedigk, S. D. Simon, R.E. Pearce, L.D. Bradford, M.J. Kennedy, J.S. Leeder, The CYP2D6 activity score: translating genotype information into a qualitative measure of phenotype, Clin. Pharmacol. Ther., 83 (2008) 234-242

[16] T.A. van der Hoeven and M.J. Coon, Preparation and properties of partially purified cytochrome P-450 and reduced nicotinamide adenine dinucleotide phosphate-cytochrome P450 reductase from rabbit liver microsomes, J. Biol. Chem., 249 (1974) 6302-6310

[17] O.H. Lowry, N.J. Rosebrough, A.L. Farr, R.J. Randall, Protein measurement with Folin phenol reagent, J. Biol. Chem., 193 (1951) 265-275

[18] T. Kronbach, D. Mathys, J. Gut, T. Catin, U.A. Meyer, High-performance liquid chromatographic assays for bufuralol 1'-hydroxylase, debrisoquine 4-hydroxylase, and dextromethorphan O-demethylase in microsomes and purified cytochrome P-450 isozymes of human liver, Anal. Biochem., 162 (1987) 24-32

[19] O. Rideg, Á. Háber, L. Botz, F. Szücs, R. Várnai, A. Miseta, G.L. Kovács, Pilot study for the characterization of pharmacogenetically relevant CYP2D6, CYP2C19 and ABCB1 gene polymorphisms in the Hungarian population, Cell Biochem. Funct.; 29 (2011) 562-568

[20] M.G. Scordo, P.A. Caputi, C. D’Arrigo, G. Fava, E. Spina, Allele and genotype frequencies of CYP2C9, CYP2C19 and CYP2D6 in an Italian population, Pharmacol. Res., 50 (2004) 195-200

[21] P. Dorado,L.E.M. Penas, A. de la Rubia, A. Llerena, Relevance of CYP2D6 -1584C>G polymorphism for thioridazine:mesoridazine plasma concentration ratio in psychiatric patients, Pharmacogenomics, 10 (2009) 1083-1089

[22] A. Llerena, P. Dorado, R. Ramirez, L.R. Calzadilla, E. Peñas-Lledó, M. Álvarez, M.E. Naranjo, I. González, B. Pérez, CYP2D6 -1584C $>$ G promoter polymorphism and debrisoquine ultrarapid hydroxylation in healthy volunteers, Pharmacogenomics, 14 (2013) 1973-1977

[23] J. O’Donohue, K.A. Oien, P. Donaldson, J. Underhill, M. Clare, R.N. MacSween, P.R. Mills, Co-amoxiclav jaundice: clinical and his- tological features and HLA class II associations, Gut, 47 (2000) 717-720

[24] M. Ingelman-Sundberg, Genetic polymorphisms of cytochrome P450 2D6 (CYP2D6): clinical consequences, evolutionary aspects and functional diversity, Pharmacogenomics J., 5(1) (2005) 6-13

[25] L.K. Teh, L. Bertilsson, Pharmacogenomics of CYP2D6: molecular genetics, interethnic differences and clinical importance, Drug Metab. Pharmacokinet., 27(1) (2012) 55-67

[26] J.K. Hicks, J.J. Swen, A. Gaedigk, Challenges in CYP2D6 phenotype assignment from genotype data: a critical assessment and call for standardization, Curr. Drug Metab.,15(2) (2014) 218-232

[27] A. Gaedigk, Complexities of CYP2D6 gene analysis and interpretation, Int. Rev. Psychiatry., 25(5) (2013) 534-553

[28] R.R. Shah, R.L. Smith, Addressing phenoconversion: the Achilles' heel of personalized medicine. Br. J. Clin. Pharmacol., 79(2) (2015) 222-240 
[29] L.E. Pope, M.H. Khalil, J.E. Berg, M. Stiles, G.J. Yakatan, E.M. Sellers, Pharmacokinetics of dextromethorphan after single or multiple dosing in combination with quinidine in extensive and poor metabolizers, J. Clin. Pharmacol., 44 (2004) 1132-1142

[30] N. Gao, X. Tian, Y. Fang, J. Zhou, H. Zhang, Q. Wen, L. Jia, J. Gao, B. Sun, J. Wei, Y. Zhang, M. Cui, H. Qiao, Gene polymorphisms and contents of cytochrome P450s have only limited effects on metabolic activities in human liver microsomes, Eur. J. Pharm. Sci., 92 (2016) 86-97

[31] G. Ersoz, Z. Karasu, C. Yildiz, U.S. Akarca, G. Yuce, Y. Batur, Severe toxic hepatitis associated with amoxicillin and clavulanic acid, J. Clin. Pharm. Ther., 26 (2001) 225-229

[32] R.J. Fontana, A.O. Shakil, J.K. Greenson, I. Boyd, W.M. Lee, Acute liver failure due to amoxicillin and amoxicillin/clavulanate, Dig. Dis. Sci., 50(10) (2005) 1785-1790

[33] A. Yu, R.L. Haining, Comparative contribution to dextromethorphan metabolism by cytochrome P450 isoforms in vitro: can dextromethorphan be used as a dual probe for both CTP2D6 and CYP3A activities?, Drug Metab. Dispos., 29(11) (2001) 1514-1520

[34] www.cypalleles.ki.se/cy2d6.html (accessed on 18th August 2016) 
Table 1. Demographic data of the human organ donors

Demographic data

Donor number

Age (year)

median (min; max)

$45(13 ; 74)$

Gender

Male/female

$67 / 61$

Cause of death

Cerebral hemorrhage/ hematoma

Subarachnoid hemorrhage $\quad 42$

Subdural hemorrhage 5

Epidural hematoma $\quad 1$

Intraventricular hemorrhage 5

Ruptured cerebral aneurysm $\quad 4$

Unknown 5

$\begin{array}{ll}\text { Stroke } & 7\end{array}$

Ischemic stroke $\quad 6$

Hemorrhagic stroke $\quad 1$

$\begin{array}{lr}\text { Tumour } & 16\end{array}$

Accident 30

Car/motor/bike accident $\quad 12$

Seizure induced cerebral injury $\quad 1$

Suicide 4

Asphyxia 1

Unknown cerebral injury $\quad 12$

Unknown 13

Anamnesis

Alcohol consumption

Medication with CYP2D6

substrate/inhibitor

Medication with agent which

cause potential liver dysfunction 
Table 2. Sequences of PCR primers and probes for CYP2D6 genotyping

\begin{tabular}{lllll}
\hline & Primer & Sequence & Probe & Sequence \\
\hline CYP2D6*3 & Forward & 5'-TGGCAAGGTCCTACGC-3' & Wild & FAM-CACAGGATGACCTGGGACC-BHQ1 \\
& Reverse & 5'-TCCATCTCTGCCAGGAAG-3' & Mutant & CalRed610-CACGGATGACCTGGGACC-BHQ2 \\
CYP2D6*4 & Forward & 5'-CTTCGCCAACCACTCC-3' & Wild & FAM-CCCCA_AGACGCCC-BHQ1 \\
& Reverse & 5'-GATCACGTTGCTCACG-3' & Mutant & CalRed610-CCCCAGGACGCCC-BHQ2 \\
CYP2D6*6 & Forward & 5'-TCTCCGTGTCCACCTTG-3' & Wild & FAM-GCTGGAGCAGTGGGTGAC-BHQ1 \\
& Reverse & 5'-GCGAAGGCGGCACA-3' & Mutant & CalRed610-GCTGGAGCAGGGGTGAC-BHQ2 \\
CYP2D6*10 & Forward & 5'-TGAGGCAGGTATGGGGCTA-3' & Wild & FAM-GCACGCTACCCCACCAGGC-BHQ1 \\
& Reverse & 5'-CAGGTTGCCCAGCCC-3' & Mutant & CalRed610-GCACGCTACTCACCAGGC-BHQ2 \\
CYP2D6*41 & Forward & 5'-TGGCTGACCTGTTCTCTGC-3' & Wild & FAM-GCCGAGGGAGGAAGGGTACAG-BHQ1 \\
& Reverse & 5'-CCTGGTCAAGCCTGTGCT-3' & Mutant & CalRed610-GCCGAGGGAGAAAGGGTACAG-BHQ2 \\
\hline
\end{tabular}

FAM, CalRed610 fluorescent labelling; BHQ, black hole quencher 
Table 3. CYP2D6 allele frequencies in Hungarian organ donors $(\mathrm{n}=128)$ and in Caucasian populations

\begin{tabular}{cccc}
\hline $\begin{array}{c}\text { CYP2D6 } \\
\text { allele }\end{array}$ & Number & \multicolumn{2}{c}{ Allele frequency (\%) } \\
Donors & Caucasians ${ }^{\#}$ \\
\hline$* 3$ & 2 & 0.8 & 2 \\
$* 4$ & 56 & 21.9 & $20-25$ \\
$* 5$ & 2 & 0.8 & $2-4$ \\
$* 6$ & 7 & 2.7 & 1 \\
$* 10$ & 9 & 3.5 & $1-2$ \\
$* 41$ & 20 & 7.8 & $7-10$ \\
Duplication & 51 & 19.9 & $2-5$ \\
\hline
\end{tabular}

\#Based on allele frequencies reported by Sachse et al. [9], Ingelman-Sundberg [24] and www.cypalleles.ki.se/cyp2d6.htm [34] 


\section{Figure legends}

Figure 1. Frequency distribution of hepatic dextromethorphan O-demethylase activity selective for CYP2D6 in human organ donors

Figure 2. Hepatic CYP2D6 activity (dextromethorphan O-demethylase) in subjects carrying various CYP2D6 genotypes

A acceptable prediction from genotype, $\nabla$ underestimated phenotype from genotype, $\square$ overestimated phenotype from genotype, $\times$ chronic alcohol consumption, + subjects under CYP2D6 substrate drug therapy, $\triangle$ subjects under Augmentin therapy

nf non-functional allele, red reduced-function allele 
Figure 1.

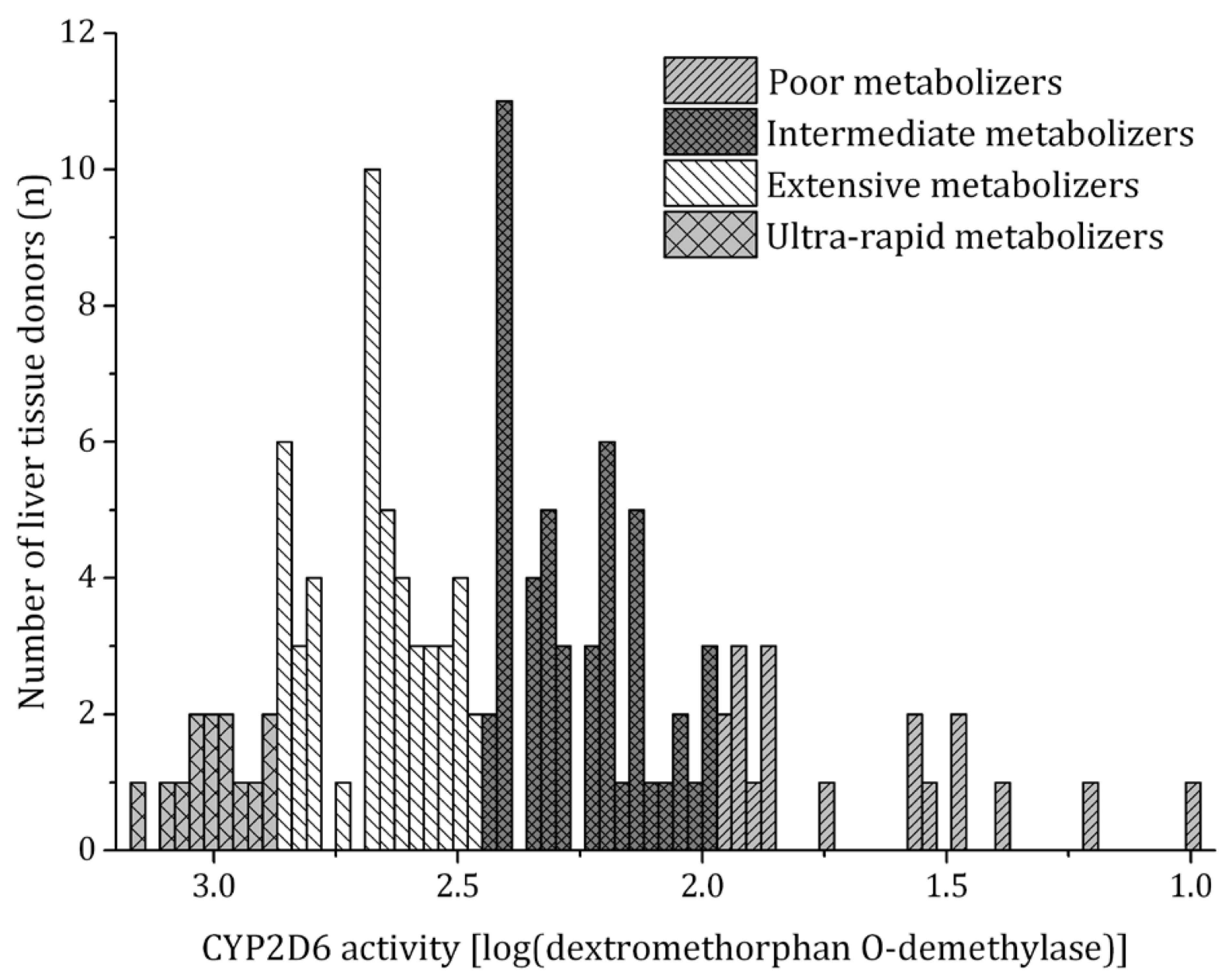


Figure 2.

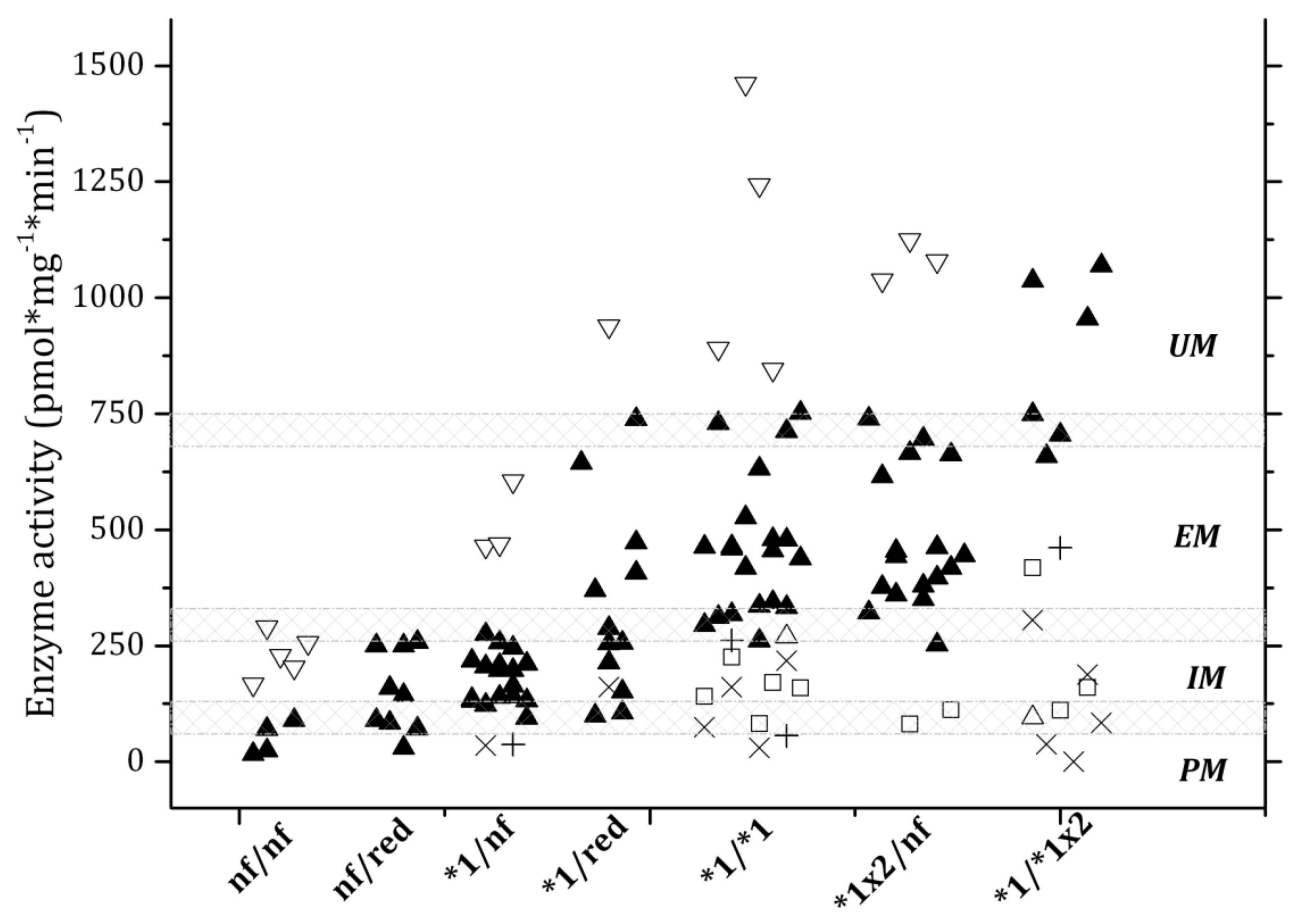

